\title{
La motricidad en el triatlón, otra mirada desde la física hacia la biomecánica*
}

\author{
[Artículos]
}

\author{
Ana Margarita Torres Aguila**
}

Recibido: 11 de noviembre de 2020

Aceptado: o9 de febrero de 2021

Citar como:

Torres-Aguila, A. M. (2021) La motricidad en el triatlón otra mirada desde la física hacia la biomecánica. Revista de Investigación Cuerpo, Cultura y Movimiento, 11(1). https://doi.org/10.15332/2422474X.6471

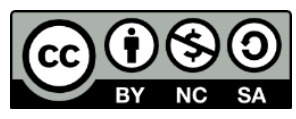

\section{Resumen}

Este artículo es una investigación científica que aborda aspectos de la motricidad, sustentada por los fundamentos de la biomecánica con un razonamiento físico como ciencias que estudian el movimiento del hombre en la actividad deportiva. La comprensión de estos conocimientos incrementa el interés por la física en los estudiantesatletas de las escuelas deportivas. Para abordar este tema, se propone un sistema de talleres apoyado con las nuevas tecnologías de la información y las telecomunicaciones (TIC).

\footnotetext{
* Artículo de investigación, educación superior, Cienfuegos, Cuba, Universidad de Cienfuegos "Carlos Rafael Rodríguez", la investigación pertenece al proyecto territorial de desarrollo local titulado: Interacción Universidad-Sociedad con perspectiva intersectorial del deporte y la salud para el desarrollo del hombre.

** Magíster, Instructora y profesora de Biomecánica, Universidad de Cienfuegos, Cuba. Correo electrónico: atorres@ucf.edu.cu; ORCID: https://orcid.org/0000-0002-0029$\underline{7380}$
} 
Palabras clave: biomecánica, habilidades motrices, mecánica

\section{Motor skills in triathlon, another look from physics to biomechanics}

\section{Abstract}

The article is a scientific research that addresses aspects of motor skills, supported by the fundamentals of biomechanics with a physical reasoning as sciences that study the movement of man in sports activity. The understanding of this knowledge increases the interest in physics in student-athletes of sports schools. To address this topic, a system of workshops supported with new information and telecommunications technologies (ICT) is proposed.

Keywords: biomechanics, motor skills, mechanics.

\section{Introducción}

La aplicación de la ciencia establece nuevas vías para lograr conocimientos más complejos y crear una nueva visión del mundo que nos rodea a partir de los principales conceptos, fenómenos y leyes de la física relacionadas a diferentes ámbitos fundamentales en la sociedad, entre ellos, el contexto deportivo. Esta realidad posibilita un cambio de pensamiento que considera la unión de elementos cognitivos desde la teoría hacia la práctica, como un elemento facilitador para la formación más sólida de una cultura general integral en los estudiantes-atletas de las escuelas de iniciación deportiva.

En Cuba, estas escuelas son consideradas como centros especializados para la atención a los estudiantes-atletas que se destacan por sus aptitudes deportivas desde una edad temprana; algunas de las escuelas destacadas son las Escuelas de Iniciación Deportiva Escolar (EIDE) y las Escuelas de Superación de Perfeccionamiento Atlético (ESPA) (Longa, 2015). 
Una preocupación de los profesores de este tipo de escuela es el poco interés que muestran los estudiantes por las asignaturas de ciencias, en particular, de la física; por esto, se requiere de una enseñanza innovadora en función de hallar soluciones más prácticas en relación con el contexto de los estudiantes. Para lograr tal propósito resulta interesante la utilización de ciencias particulares aplicadas al deporte, donde la biomecánica deportiva desarrolla un rol fundamental por su relación con la física y la posibilidad de integrar los conocimientos de los estudiantesatletas desde sus vivencias deportivas. Con esta relación se haría posible la correspondencia interdisciplinar al vincular los conceptos, fenómenos y leyes de la física que sean relevantes para la explicación del movimiento del hombre en la actividad deportiva.

Para Gómez et ál. (2016), Pilotos y Morejón (2018) y Estrada (2018) los estudios biomecánicos se han hecho un eslabón imprescindible por muchas razones, entre ellas, la importancia de los resultados deportivos en diversos ámbitos de la vida y la dificultad, cada vez mayor, para mejorar las marcas, pero principalmente como prevención de lesiones. Esto denota la necesidad del perfeccionamiento de la didáctica de las ciencias.

En Cuba también se ha llevado a cabo el perfeccionamiento de la didáctica y ciencias aplicadas al deporte, al integrar resultados científicos expresados en tesis de maestrías y doctorados con el objetivo de analizar situaciones deportivas a través de estudios biomecánicos en los estudiantes-atletas de alto rendimiento. Estos análisis biomecánicos se sustentan por los conceptos, leyes y principios físicos que refieren varios autores, entre ellos tenemos a González et ál. (2016), Perdomo et ál. (2018), Perdomo (2018) y Ramírez (2018).

Los autores tratan las características cinemáticas y dinámicas del hombre en la actividad física deportiva para así analizar la técnica deportiva en este tipo de escuela (EIDE). La limitación principal de estos estudios radica en 
que se analizan los deportes de alto rendimiento sin tener en cuenta el proceso desde la iniciación deportiva, por lo que no se socializan estas investigaciones en la docencia de las escuelas deportivas. Como elemento a destacar, estos autores afirman que la biomecánica deportiva permite desarrollar una investigación encaminada a establecer ejercicios especiales dirigidos al perfeccionamiento de la acción motora. Por otra parte, en estas investigaciones se analiza y se llega a un mayor entendimiento acerca de las habilidades motrices y del aprendizaje motor, que se trata de analizar detalladamente los gestos deportivos y las técnicas que están presentes en las acciones rápidas y de corta duración que a simple vista son difíciles de observar.

En general, se evidencian soluciones a problemáticas relacionadas con el perfeccionamiento en los deportes de alto rendimiento, sin embargo, existen insuficiencias al momento de relacionar las experiencias deportivas de los estudiantes-atletas con la enseñanza de la física, lo que incide en la falta de interés por la asignatura. Otro aspecto importante, es la complejidad de dictar clases de biomecánica deportiva en la solución de problemas desde el punto de vista de la física, pues los estudiantes no tienen un conocimiento sólido sobre física. Por lo anterior, se hace necesario perfeccionar la didáctica para modificar el interés por la asignatura de física y comprender las habilidades motrices en la ejecución del movimiento. De ahí que el objetivo de la investigación esté encaminado a la elaboración de talleres que eleven el interés por la física con el estudio de las habilidades motrices desde la biomecánica deportiva en las escuelas deportivas.

\section{Los fundamentos de la física en la rama de la mecánica y las habilidades motrices del triatlón desde la biomecánica}

Estrada (2018) afirma que los conceptos, leyes y principios de la física en la rama de la mecánica pueden usarse para comprender la técnica 
deportiva con el empleo de estudios biomecánicos y las ecuaciones físicas y matemáticas. Abordar su estudio implica la necesidad de relacionarse con las ciencias aplicadas al deporte para un mismo fin, el entrenamiento deportivo y la calidad de los estudiantes-atletas. Sin embargo, si bien en un principio esto puede entenderse como una dificultad, una vez que se dominan los conceptos fundamentales de la física mecánica, su aplicación en la actividad deportiva contribuye en la elección profesional y su accionar diario.

Para su estudio, la mecánica se puede dividir en tres grandes ramas: la cinemática, que estudia el movimiento de los cuerpos en el espacio con un carácter meramente descriptivo, sin detenerse a evaluar las causas productoras, ni el gasto energético demandado. La cinética o dinámica, que estudia las causas productoras de los movimientos articulares; y la estática, que estudia el diseño de las estructuras, y la respuesta de estas ante las cargas aplicadas (Perdomo, 2018).

Gómez et ál. (2016) plantean que la mecánica del movimiento es la variación objetiva de la posición de un cuerpo dentro del espacio en que se desenvuelve y en un determinado periodo de tiempo. Por lo tanto, para que el desplazamiento sea objeto de estudio, debe existir un sistema de referencia para determinar el cambio de posición de un cuerpo dentro del espacio. En el externo se utiliza el sistema de coordenada, y en el interno, la medición de ángulos entre segmentos adyacentes de la cadena.

Es importante destacar que la producción del movimiento es provocada por la fuerza, en cuya estructura actúan dos efectos. Cuando es un efecto externo existe el cambio del estado físico en que el cuerpo se encuentra; y cuando es interno representa la estructura molecular del cuerpo, creando un estado de tensión que se manifiesta con deformación y aumento de temperatura.

Revista de Investigación Cuerpo, Cultura y Movimiento

ISSN: 2248-4418 | e-ISSN: 2422-474X | DOI: https://doi.org/10.15332/2422474X

Vol. 11 N.o 1 | enero-junio de 2021 
Según Ramírez (2018), la cinemática es el área de la mecánica que centra su estudio en el movimiento de los cuerpos en el espacio con un carácter descriptivo, es decir, no evalúa las causas que los producen, ni el gasto energético demandado para su realización. Para ello, existen consideraciones en cuanto la masa del cuerpo (centro de masa) con proyecciones al eje mecánico. Todas las magnitudes mecánicas pueden expresarse mediante la combinación de tres patrones primarios, como longitud, tiempo, y masa.

En el deporte del triatlón se comienza de los 10 a los 13 años; se da una iniciación a las habilidades y tareas específicas que tienen un carácter lúdico-deportivo y se refieren a actividades deportivas o actividades expresivas. En este se trabajan habilidades genéricas que son comunes a muchos deportes. Se inician habilidades específicas de cada deporte y técnicas para mejorar los gestos. De los 14 a los 17 años se incluye el desarrollo de habilidades motrices y la trasformación escalonada a las capacidades motrices específicas, la iniciación a la especialización deportiva y el trabajo de técnica y táctica con aplicación real.

Pilotos y Morejón (2018) enuncian que, desde el punto de vista biomecánico, el cuerpo humano constituye un sistema, es decir, un conjunto de elementos interrelacionados con un fin común de generar la movilidad necesaria para sobrevivir y dejar descendencia. Como todo sistema, puede subdividirse para su estudio en subsistemas, que en este caso los constituyen las llamadas cadenas biocinemáticas. Estas constituyen la unidad dinámico-funcional del sistema y están conformadas por sucesivas cadenas óseas y las unidades biomecánicas que las unen. Es una incógnita el entendimiento de este tipo de deporte para atletas que se inician por la secuencia y trasferencia de los gestos deportivos y la técnica. La cadena biocinemática se va trasformando según sus gestos deportivos, abierta, ramificada o cerrada. Esta unión sucesiva de varios 
pares biocinemáticos son los que rigen la transferencia con las magnitudes físicas de velocidad, tiempo, fuerza, energía, entre otros.

Para Ruíz (1987), citado en Falcón y Rivero (2010), los procesos humanos llevan implícitas las acciones pedagógicas y los resultados del aprendizaje. Se identifican como mediadores, por un lado, las actividades motrices específicas para alcanzar el objetivo y, por otro lado, la actividad cognitiva y motivacional relacionada con la actividad que se va a realizar. Estos mediadores permiten declarar para su funcionamiento las características de los estudiantes en tres ámbitos: el cognitivo, el motor y el afectivo. En esta investigación se trabaja un ámbito afectivo, donde los aspectos de interés y de sensibilidad cuentan para activar el aprendizaje hacia la práctica deportiva desde la física.

El desarrollo de las habilidades motrices presupone un reto cada vez mayor para tributar a la educación integral y fortalecer aquellos aspectos cognoscitivos y afectivos que integran la unidad del ser humano; de esta manera, se podrá alcanzar un mayor nivel de resultados de aprendizajes, que propicie la reflexión y el debate desde las estrategias cognoscitivas de los estudiantes-atletas. Para esto, hay que destacar la influencia que tienen las capacidades motrices para el desarrollo de las habilidades propias.

Se hace un análisis de las capacidades motrices que se deben fortalecer en los estudiantes-atletas de triatlón. La velocidad de un cuerpo puede mantenerse constante o registrar cambios que la incrementen o disminuyan. Dependiendo de ello, los movimientos se clasificarán como movimiento rectilíneo uniforme o movimiento rectilíneo uniformemente variado. En un movimiento uniforme, el cuerpo recorre iguales distancias por unidad de tiempo. En la asignatura de biomecánica deportiva se denomina rapidez.

Para los investigadores González et ál. (2016) y Pilotos y Morejon (2018) la rapidez constituye la relación entre la distancia recorrida en una unidad de 
tiempo. La aceleración constituye la variación de la velocidad en determinado periodo. Para que un cuerpo sea acelerado necesita de una fuerza que lo acelere, debido a que la aceleración a la que es sometido un cuerpo es resistida por la inercia propia de este, ya que esta tiene una relación directa con la masa que lo constituye; la masa va a estar en relación inversa a la aceleración $(\mathrm{a}=\mathrm{F} / \mathrm{m})$. La aceleración se mide en $\mathrm{m} / \mathrm{s}^{2}$. Este despeje proviene de la ecuación física $\mathrm{F}=\mathrm{m}$. a, segunda ley de Newton.

Según Sánchez (1984), citado en Oliva (2015), por habilidades motrices básicas entendemos aquellos actos motores que se llevan a cabo de forma natural y que constituyen la estructura sensomotora básica y el soporte del resto de las acciones motrices que el ser humano desarrolla. Además, plantea que el desarrollo de las habilidades motrices entre los 14 y los 17 años incluye el desarrollo de habilidades motrices específicas, la iniciación a la especialización deportiva y el trabajo de técnica y táctica con aplicación real.

En esta investigación se profundiza en la motricidad gruesa; se evidencia desde las categorías escolares la necesidad de fortalecer este tipo de motricidad en el deporte de triatlón, ya que se desarrollan en la resistencia, la fuerza y la velocidad, por las carreras prolongadas y la trayectoria amplia y compleja de sus transiciones. El triatlón es un deporte multidisciplinar que incluye natación, ciclismo, carrera y las transiciones. El movimiento de cada disciplina desempeña un papel vital en el rendimiento del atleta, donde la técnica en la ejecución deportiva exige el dominio de una serie de habilidades específicas para cada segmento del triatlón. Si estas no se cumplen, afectará negativamente el rendimiento general de la prueba.

Según Donskoi y Zatsiorski (1988), citado en Perdomo (2018), entre las disciplinas de mayor dificultad se encuentra el ciclismo, pues sus 
capacidades motoras son la resistencia, la fuerza, la flexibilidad, la coordinación y la velocidad. En la investigación se hace un análisis de las habilidades motrices de incidencia en el deporte de estudio.

La fuerza es la capacidad para vencer o contrapesar una resistencia (un peso) mediante la actividad muscular. El entrenamiento busca desarrollar diferentes cualidades, como incrementar la masa muscular, reforzar los tejidos conjuntivos y de apoyo y mejorar la constitución corporal. Todo esto, sin olvidar la óptima relación entre fuerza y técnica deportiva; si se desarrolla no se llegará a la fatiga muscular.

Cuando la fuerza se vincula con la resistencia presentan un régimen isotónico (o dinámico), que es cuando la tensión provoca un cambio de longitud de los músculos. Este puede ser de tipo concéntrico, que es cuando se disminuye la longitud, es decir, disminuye la longitud de las inserciones musculares y su función es agonista; mientras que el excéntrico es en el que aumenta la longitud de las inserciones y su función es antagonista. En este deporte de largas distancias está presente la fuerza y la resistencia, por lo que es necesario incrementar la capacidad de la fuerza durante el mayor tiempo posible para disminuir las lesiones musculares.

Otra capacidad es la flexibilidad; el déficit de flexibilidad puede complicar la asimilación de las habilidades motrices. Los estudios biomecánicos del triatlón evidencian la necesidad de fortalecer la capacidad de flexibilidad de la articulación coxofemoral y tibiotarsiana sobre el rendimiento de un ciclista entre 15 a 20 kilómetros contrarreloj y en el kilómetro con partida detenida. En este caso, son inversamente proporcionales la longitud de la articulación coxofemoral con el tiempo. Por ejemplo, para mejorar las amplitudes de movimiento de la articulación se necesita incrementar las sesiones de entrenamiento ( +40 sesiones), cada una de media hora, mientras que si se deja de entrenar una sola semana se puede perder. El 
conocimiento previo de cómo ejercitar la flexibilidad disminuye el riesgo de las lesiones, ya que se fortalece las zonas vulnerables.

\section{Metodología}

Para la investigación se seleccionó una muestra que coincide con la población de 60 estudiantes-atletas de la escuela de iniciación deportiva escolar, Jorge Agostini Villasana de la provincia Cienfuegos, que pertenecen al deporte triatlón.

El diseño de investigación aplicado fue un enfoque cualitativo, diseño no experimental, transeccional descriptivo; este profundiza en los estudios biomecánicos del triatlón con el empleo de videos y el software Kinovea para analizar la técnica deportiva y evitar el riesgo de lesiones. La utilización de métodos científicos como la observación directa, la encuesta y la entrevista, posibilitaron mejorar la propuesta en el estudio de profundización de la situación en la práctica.

La propuesta de talleres está dirigida a los estudiantes-atletas en función de que estos realicen análisis profundos de los contenidos de la física mecánica y se tome como muestra el triatlón, atendiendo a las inquietudes y falta de conocimiento de este deporte. Para su entendimiento se utilizaron talleres para profundizar la temática y se analizó la preparación técnica de los estudiantes-atletas de triatlón para evitar lesiones. Se mostraron resultados de estudios realizados y se propició el intercambio con los profesores de ciencias y entrenadores deportivos de la institución seleccionada sobre la temática propuesta (ventajas, objetivos propuesta, actividades a desarrollar y contenidos fundamentales). Se escucharon las sugerencias para lograr un desarrollo exitoso de las acciones.

Revista de Investigación Cuerpo, Cultura y Movimiento

ISSN: 2248-4418 | e-ISSN: 2422-474X | DOI: https://doi.org/10.15332/2422474X

Vol. 11 N.o 1 | enero-junio de 2021 


\section{Sistemas de talleres}

A continuación, se presentan los diferentes talleres que se elaboraron para elevar el interés por la física con el estudio de las habilidades motrices desde la biomecánica deportiva en las escuelas deportivas. Estos talleres se imparten semanalmente.

En el primer taller se realizó un intercambio de saberes, se abordan las mejores prácticas y experiencias en el tratamiento de leyes, conceptos y principios físicos de la física mecánica, que tuvo como objetivo valorar los fundamentos biomecánicos y sus relaciones con la física, sus posibilidades con el uso de las TIC y su gran aplicación en el entrenamiento deportivo de triatlón.

La actividad incitó a los alumnos al estudio cinemático y dinámico en la traslación y rotación del movimiento. Esto puso sobre la mesa las reflexiones acerca de la importancia y actualidad de la aplicación de este conocimiento en la práctica cotidiana del triatlón, que sirvieron para evidenciar el interés de los participantes en el tema, algunos expusieron su desconocimiento al respecto. Además, señalan la necesidad de su aplicación en los entrenamientos, se enfatiza en lo apropiado del uso de las TIC y el software en estos estudios y la posibilidad de su aplicación, sin el empleo de grandes recursos, en la revisión de artículos en Internet.

El segundo taller abre paso al acercamiento a la biomecánica deportiva, que tuvo como objetivo valorar el surgimiento, actualidad y perspectivas de la asignatura y la estrecha relación que tiene con la física. Esto se llevó a cabo con el uso de las TIC y su gran aplicación en el entrenamiento deportivo del triatlón.

El contenido de la enseñanza del taller realizado fue el surgimiento, actualidad y perspectivas de la biomecánica deportiva; el hombre como sistema biomecánico, sustentado por conceptos, leyes y principios físicos; 
el carácter interdisciplinario y el valor de los métodos biomecánicos de investigación; y las habilidades motrices de las transiciones del deporte triatlón.

El tercer taller se estudian las características biomecánicas que se manifiestan en los movimientos humanos durante la actividad deportiva y el uso de las TIC para el análisis del triatlón. Este tuvo como objetivo caracterizar los movimientos de traslación y rotación, así como el procedimiento para el análisis biomecánico de los movimientos, teniendo en cuenta la aplicación del software en el análisis de ejecuciones técnicas de la práctica deportiva.

Los contenidos tratados fueron, las características cinemáticas del movimiento, ecuaciones y gráficos, los movimientos de traslación y rotación, el uso y las ventajas de las TIC para el análisis de los movimientos deportivos; con este se le da tratamiento a las invariantes, principios, leyes y conceptos de la mecánica que rigen la motricidad humana y la biomecánica como ciencia, métodos, procedimientos de trabajo, técnicas de investigación biomecánica y aplicación de las TIC para la obtención de características cinemáticas y dinámicas del movimiento humano.

En el cuarto taller se realiza un estudio de las capacidades motrices en el triatlón por la observación de videos de atletas con maestría deportiva del deporte, analizando y usando el software para comprobar su factibilidad en el análisis de los movimientos humanos; su objetivo fue aplicar el software informático a los métodos y técnicas de carácter biomecánicos que posibilitaron estudiar el movimiento humano y los indicadores propios de su técnica deportiva.

Los contenidos abordados fueron, la caracterización del proceso de filmación, movimientos en los ejes y los planos, simulación con el velocímetro y ejemplificación del software Kinovea; esto se llevó a cabo

Revista de Investigación Cuerpo, Cultura y Movimiento

ISSN: 2248-4418 | e-ISSN: 2422-474X | DOI: https://doi.org/10.15332/2422474X

Vol. 11 N.o 1 | enero-junio de 2021 
utilizado el análisis biomecánico de los movimientos humanos y determinando los indicadores a partir de la aplicación del análisis de videos en acciones motoras, con el objetivo de analizar las características cinemáticas y dinámicas del movimiento humano y las habilidades motrices.

\section{Resultados y discusión}

Con la aplicación del software Kinovea y el análisis de videos deportivos, pudo profundizarse en la ejecución de los movimientos de los atletas en este tipo de deporte en la determinación de variables como la trayectoria, la postura, el centro de masa, la aceleración, la velocidad, los ángulos, la fuerza, las fases, entre otras.

En la tabla 1, donde se representa la aceptación de los talleres en los estudiantes-atletas, se evidencia un incremento considerable del interés por la física mediante el estudio biomecánico de las capacidades motrices.

Tabla 1. Nivel de aceptación de talleres en los estudiantes atletas

\begin{tabular}{|l|r|r|r|r|r|}
\multicolumn{1}{|c|}{ Talleres } & \multicolumn{1}{c|}{ Antes } & \multicolumn{1}{c|}{$\%$} & \multicolumn{1}{c|}{ Después } & \multicolumn{1}{c|}{$\%$} \\
\hline Primer taller & 25 & 41 & 37 & 61 \\
\hline Segundo taller & 38 & 63 & 42 & 70 \\
\hline Tercer taller & 46 & 76 & 54 & 90 \\
\hline Cuarto taller & 55 & 91 & 60 & 100 \\
\hline
\end{tabular}

Nota: En la tabla se representa los resultados de la encuesta "interés por conocer las capacidades motrices desde la biomecánica deportiva con su relación de leyes, conceptos y principios físicos". Su realización fue en dos etapas (antes y después) de los talleres.

Fuente: elaboración propia.

Con la puesta en práctica de la propuesta de los talleres, se hizo un análisis cualitativo del conocimiento de las habilidades motrices desde la biomecánica deportiva del triatlón y su relación con conceptos, leyes y 
principios físicos. Con la estrategia cognoscitiva antes expuesta se consolidaron los contenidos de cinemática, dinámica, energía, centro de gravedad, entre otros, que logró un mayor interés por la asignatura de física. Se evidencia una mayor comprensión por parte de los estudiantesatletas de la asignatura y esta tuvo un gran impacto en el mejoramiento de las técnicas de transición motora y las reacciones rápidas en la ejecución y los gestos deportivos.

Para constatar el incremento del interés por la temática planteada se utiliza la técnica "Positivo, Negativo e Interesante":

Se logran afirmaciones positivas del sistema de talleres para los estudiantes-atletas al considerar que la física mecánica es importante para la sociedad; aluden la necesidad de integrar otras ciencias para estudiar el movimiento mecánico del hombre y la motricidad en la actividad física deportiva; refieren que los estudios biomecánicos mediante los softwares y videos presentan utilidad para analizar la técnica deportiva; la amplitud de potencialidades hacia el mejoramiento deportivo; manifiestan no conocían esto con claridad

Se hace necesario seguir perfeccionando el sistema de talleres para los estudiantes-atletas, ya que se evidencian planteamientos negativos que se pueden modificar al señalar que se debe evaluar el horario en que se desarrolló, pero no había otro; debió durar más tiempo, plantean que existen carencias de medios y tecnologías para su realización periódica y cotidiana. Otros aseguran que les gustaría saber cómo implementar la temática, más elementos y disponer de mayor tiempo para su aplicación. Lo interesante del sistema de talleres es que logró el objetivo de elevar el interés por la física en los estudiantes-atletas; comprendieron la necesidad de estudiar el organismo del hombre en su interior para así conocer su cuerpo cuando se ejecuta algún movimiento en el entrenamiento deportivo; refieren que el estudio biomecánico a través del video soluciona 
y erradica malas técnicas y, por consiguiente, se evita el riesgo de lesiones. Afirman que con la aplicación de softwares se estudian las fases de la ejecución de la técnica de los deportes, por su complejidad, como el triatlón.

Los resultados más relevantes presuponen el alcance que tienen estas ciencias aplicadas en el entrenamiento deportivo; conocer que no es necesario una tecnología tan sofisticada para empezar a aplicarla, solo se debe tener recursos disponibles para su uso; las destrezas que desarrollan los estudiantes-atletas por la elección profesional hacia la carrera de Cultura física, se propició en ambiente adecuado, un clima favorable que se mantuvo en el desarrollo del sistema de talleres.

\section{Conclusiones}

Con la investigación se determinaron los fundamentos teóricometodológicos que sustentan la problemática del interés de los estudiantes-atletas por las capacidades motrices desde la biomecánica deportiva y su relación con las leyes, conceptos y principios físicos. Se generó mayor comprensión acerca del tema por su importancia para mejorar el rendimiento deportivo y evitar el riesgo de lesiones. Los videos y análisis biomecánicos de diferentes atletas de triatlón a nivel nacional e internacional conllevan un aprendizaje cognoscitivo complejo y significativo para los estudiantes-atletas de este tipo de deporte.

\section{Referencias}

Estrada, Y.C (2018). Biomecánica: De la física mecánica al análisis de gestos deportivos. 48. Universidad Santo Tomás. Ediciones USTA. ISBN: 978-958-782-132-1. https://repository.usta.edu.co/bitstream/handle/11634/12464/Obracompleta.201 8Estradayisel.pdf?sequence $=1$ \&isAllowed $=\mathrm{y}$ 
Falcón, V y Rivero, E. (2010). Aprendizaje motor. Las habilidades motrices básicas: coordinación y equilibrio. Revista Buenos Aires, 15(147), 1-5. https://gse.com/uploads/blog adjuntos/aprendizaje motor. las habilidades motrices b sicas coordinaci $n$ y equilibrio.pdf

Gómez, A. P., Ramírez, S., Infante, N.M., Pavón, J.L. y Pozo, R.J. (2016). La Biomecánica al servicio de la actividad física en Holguín. Revista científica especializada en Ciencias de la Cultura Física y del Deporte, 16(42), 89-102. https://deporvida.uho.edu.cu/index.php/deporvida/article/view/569

Longa-Martínez, T. (2015). El aprendizaje significativo de la física para estudiantesatletas de las escuelas deportivas. Rastros Rostros, 17(31).

http://dx.doi.org/10.16925/ra.v17i31.1093

Oliva, J. (2015). Motricidad, Psicomotricidad, nadar, Natación y su relación con la educación física. [Diapositiva de Prezi].

https://prezi.com/wfehtriqgqyi/motricidad-psicomotricidad-nadar-natacion-y-surelacion-c/

Perdomo, J. M, Pegudo, A. G. y Capote, T. E. (2018). Premisas para la investigación biomecánica en la cultura física. Revista Cubana Educación Superior, 37(2), 104114. http://scielo.sld.cu/scielo.php?script=sci arttext\&pid=S025743142018000200008

Perdomo, J. M (2018). La superación profesional de entrenadores deportivos de Villa Clara en la aplicación de la Biomecánica [Tesis Doctoral]. Universidad Central Marta Abreu de las Villas, Cuba.

Ramírez, Y. (2018). Preparación de profesores de Física en preuniversitario para la interdisciplinariedad en el proceso de enseñanza aprendizaje. [Tesis Doctoral]. Universidad Central "Marta Abreu de las Villas, Cuba.

Revista de Investigación Cuerpo, Cultura y Movimiento

ISSN: 2248-4418 | e-ISSN: 2422-474X | DOI: https://doi.org/10.15332/2422474X

Vol. 11 N.o 1 | enero-junio de 2021 